\title{
Noncoherent Multi-Way Relay Based on Fast Frequency-Hopping $M$-ary Frequency-Shift Keying
}

\author{
Jianfei Cao ${ }^{\# 1}$, Lie-Liang Yang ${ }^{* 2}$, Zhangdui Zhong \#3 \\ \# State Key Lab of Rail Traffic Control and Safety \\ Beijing Jiaotong University, Beijing 100044, China \\ 1 jc09v@ecs. soton.ac.uk, ${ }^{3}$ zhdzhong@bjtu.edu. cn \\ * School of Electonics and Computer Science \\ University of Southampton, SO17 1BJ, United Kingdom \\ ${ }^{2}$ liydecs.soton.ac.uk
}

\begin{abstract}
Information exchange among a group of users is implemented with the aid of fast frequency-hopping $M$-ary frequency-shift keying multi-way relay (FFH/MFSK MWR). The FFH/MFSK MWR scheme uses two time-slots per symbol for achieving the information exchange, regardless of the number of users involved. During the first time-slot, all the users communicate with a relay based on the FFH/MFSK principles. Then, without recovery of the symbols received, the relay forms a time-frequency (TF) matrix, which is forwarded to all the users during the second time-slot. During the second timeslot, each user receives signals from the relay and, based on which, detects the other users' information. In the FFH/MFSK MWR scheme, both the relay and the users use square-law assisted noncoherent techniques for detection. While the relay uses simple threshold detection, three types of detectors, namely the maximum likelihood multiuser detector (ML-MUD), suboptimum ML-MUD (SML-MUD) and the majority vote based single-user detector (MV-SUD), are considered for detection at the users. Finally, in this paper, the error performance of the FFH/MFSK MWR systems is investigated by simulations, when assuming communications over Rayleigh fading channels.
\end{abstract}

\section{INTRODUCTION}

The growing demand for information exchange among multiple users in wireless networks has drawn great research attention in recent years. The concept of multi-way channels for information exchange among multiple users was considered in [1] as early as in 1977, where the achievable rate region was studied. In a multi-way communication network, users are expected to share their information with each other, meaning that a user sending its messages to the other users also intends to decode the messages sent by the other users. Recently, multiway communications have been suggested to be implemented with the aid of relays, yielding the concept of multi-way relay $[2,3]$. Specifically, when only two users are involved, the multi-way relay is reduced to the well-known two-way relay [4]. Due to the broadcasting nature of wireless signals, it is usually very hard for wireless transceivers to transmit and receive signals simultaneously on the same frequency band. Hence, half-duplex is commonly used. In this case, even for the two-way relay scheme, the time, frequency or space resource required will at least be doubled in comparison with that required by the one-way scheme. When multi-way relay is considered, further resource is explicitly demanded, which might become extreme, when without elaborate design of the information exchange protocols.

In order to implement high-efficiency information exchange among a group of users, in this paper, we propose a lowcomplexity multi-way relay scheme based on fast frequencyhopping and $M$-ary frequency-shift keying, which we refer to as the FFH/MFSK MWR scheme. No matter how many users are involved, our FFH/MFSK MWR scheme uses only two time-slots per symbol period for information exchange, which is achieved with the aid of one relay. During the first time-slot, all the users send their information to the relay through their multiple-access channels. At the relay, the received TF signals are noncoherently processed to form a hard-decision TF matrix, which is broadcasted by the relay to all the users during the second time-slot. Finally, each of the users involved detects the other users' information also based on noncoherent approaches. One typical application of the proposed FFH/MFSK MWR is for information exchange among a group of users which cooperate with each other. In this case, the FFH/MFSK MWR system may be operated either in the frequency band of the main system operated or in the frequency-bands detected in real-time fashion based on cognitive radio principles.

One of the typical characteristics of the proposed FFH/MFSK MWR scheme is the noncoherent processing, which demands only low-complexity techniques. By contrast, most previous techniques proposed in literature require coherent processing based on accurate and instantaneous channel state information (CSI). Hence, their complexity may be very high, especially, in relay networks. In the context of the relay networks using noncoherent processing, [5] has developed a near optimum detector for MFSK signalling, which does not require CSI but requires the second order statistics of the channels as well as the decoding error probability at relays. In [6], optimum noncoherent detection for amplify-and-forward (AF) relay networks has been addressed, and the lower as well as upper bounds for the achievable bit error rate (BER) have been derived. Additionally, in [7,8], the authors have studied the detection based on the generalized likelihood ratio test (GLRT) for both AF and decode-and-forward (DF) relay systems.

The FFH/MFSK has been widely studied in the context of multi-access communications, as shown, e.g., in [9-14]. In addition to its relatively low complexity, the FFH/MFSK also has the advantages of, such as, inherent frequency diversity, effectiveness for combating various types of interference, no requirement of successive frequency bands, etc. For noncoherent detection in FFH/MFSK systems, there are a range of SUD and MUD algorithms proposed for the conventional multiple-access communications [9-14]. In this paper, three types of them, namely, the ML-MUD, SML-MUD and MVSUD, are extended for achieving noncoherent detection in our FFH/MFSK MWR systems. Furthermore, the performance 
of the FFH/MFSK MWR systems employing the abovementioned detectors is investigated and compared.

\section{SySTEM MODEL}

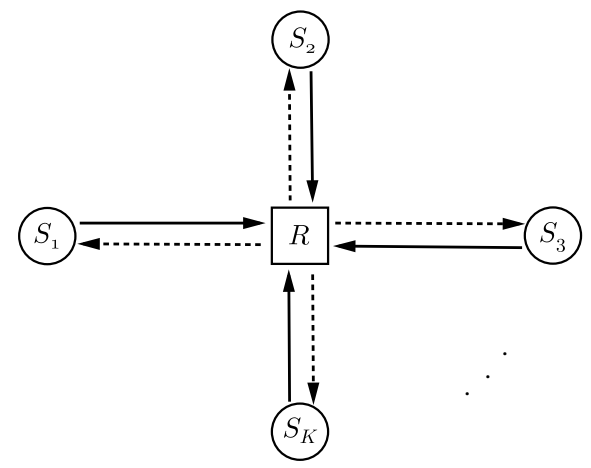

Fig. 1. Paradigm of multi-way relaying schemes, where the solid and dashed lines represent the multi-access and broadcasting channels, respectively.

In this section, we introduce the principles of our proposed FFH/MFSK MWR scheme, which uses one relay to serve all the other users. The schematic diagram of the FFH/MFSK MWR is shown in Fig. 1, where one relay $R$ is located at the center of $K$ users exchanging information. The system is operated in two phases (or two time-slots), multiple-access and broadcasting, based on half-duplex. During the multiaccess phase, all the $K$ users transmit synchronously their FFH/MFSK signals to the relay. The equivalent complex lowpass signal transmitted by user $k, k \in\{1, \ldots, K\}$, can be formulated as

$$
S_{k}(t)=\sqrt{2 P} \sum_{l=0}^{L-1} \psi_{T_{h}}\left(t-l T_{h}\right) \exp \left(j 2 \pi f_{l}^{(k)} t+\varphi_{l}^{(k)}\right)
$$

where $P$ denotes the transmission power per dimension, $L$ represents the number of time chips used for conveying one $M$-ary symbol and $T_{h}$ is the chip duration, while $\varphi_{l}^{(k)}$ denotes the random phase generated from carrier modulation. In (1), the time domain pulse-waveform $\psi_{T_{h}}(t)$ is defined within $\left[0, T_{h}\right)$, which is normalized to satisfy $\int_{0}^{T_{h}} \psi_{T_{h}}^{2}(t) d t=T_{h}$. Furthermore, in (1), the frequency $f_{l}^{(k)}$ is jointly determined by the FH address and data of user $k$ in the way as [13]

$$
\boldsymbol{y}_{k}=X_{k} \cdot \mathbf{1} \oplus \boldsymbol{a}_{k} \stackrel{\mathrm{MFSK}}{\Longrightarrow} \boldsymbol{f}^{(k)}=\left[f_{0}^{(k)}, f_{1}^{(k)}, \ldots, f_{L-1}^{(k)}\right]
$$

where $X_{k}$ denotes the $M$-ary symbol transmitted by user $k$ 1 is an all-one vector of length $L, \boldsymbol{a}_{k}=\left[a_{0}^{(k)}, a_{1}^{(k)}, \ldots, a_{L-1}^{(k)}\right]$ is the unique $\mathrm{FH}$ address assigned to user $k$, where $a_{l}^{(k)}$ is an element of the Galois Field $G F(M)$ [10], while $\oplus$ denotes the addition operation in $G F(M)$. After the MFSK modulation, in (2), the elements of $\boldsymbol{y}_{k}$ are mapped to the corresponding frequencies $\boldsymbol{f}^{(k)}=\left[f_{0}^{(k)}, f_{1}^{(k)}, \ldots, f_{L-1}^{(k)}\right]$.

From the multiple-access channels, the signal received by the centred relay is given by

$$
\begin{aligned}
R(t)=\sum_{k=1}^{K} \sum_{l=0}^{L-1} \sqrt{2 P} h_{l}^{(k)} \psi_{T_{h}}\left(t-l T_{h}\right) \\
\\
\quad \times \exp \left(j 2 \pi f_{l}^{(k)} t\right)+N(t)
\end{aligned}
$$

where $h_{l}^{(k)}$ represents the channel fading gain with unit variance, which is assumed to obey the independent Rayleigh distribution with respect to the indexes $l$ and $k, N(t)$ denotes the additive white Gaussian noise (AWGN) with single-sided power spectrum density (PSD) of $N_{0}$ per dimension. Note that, the carrier phase $\varphi_{l}^{(k)}$ seen in (1) has been absorbed into $h_{l}^{(k)}$ in (3).

Without channel estimation, the relay obtains observations from square-law detectors [15]. The normalized observation $R_{m l}$ with respect to the $m$ th, $m \in\{0, \ldots, M-1\}$, frequency tone and the $l$ th, $l \in\{0, \ldots, L-1\}$, chip can be expressed as

$$
\begin{aligned}
R_{m l} & =\left|\int_{l T_{h}}^{(l+1) T_{h}} \frac{R(t)}{\sqrt{2 P} T_{h}} \psi_{T_{h}}^{*}\left(t-l T_{h}\right) \exp \left(-j 2 \pi f_{m} t\right) d t\right|^{2} \\
& =\left|\sum_{k=1}^{K} h_{l}^{(k)} \delta\left(f_{l}^{(k)}, f_{m}\right)+N_{m l}\right|^{2}
\end{aligned}
$$

where $N_{m l}$ is the normalized Gaussian noise sample distributed with mean zero and a variance $\sigma_{r}^{2}=L N_{0} / E_{s}$, where $E_{s}=L P T_{h}$ is the energy per symbol. In (4), $\delta(a, b)$ is an indicator function defined as $\delta(a, a)=1$ and $\delta(a, b)=0$, if $a \neq b$. In (4), $\left\{h_{l}^{(k)}\right\}$ and $N_{m l}$ obey complex Gaussian distributions. Hence, it can be shown that the square-law detector's output $R_{m l}$ follows exponential distribution with a mean given by $K_{m l}+\sigma_{r}^{2}$, where $K_{m l}, K_{m l}=0,1, \ldots, K$, denotes the number of users activating the $m$ th frequency tone within the lth chip.
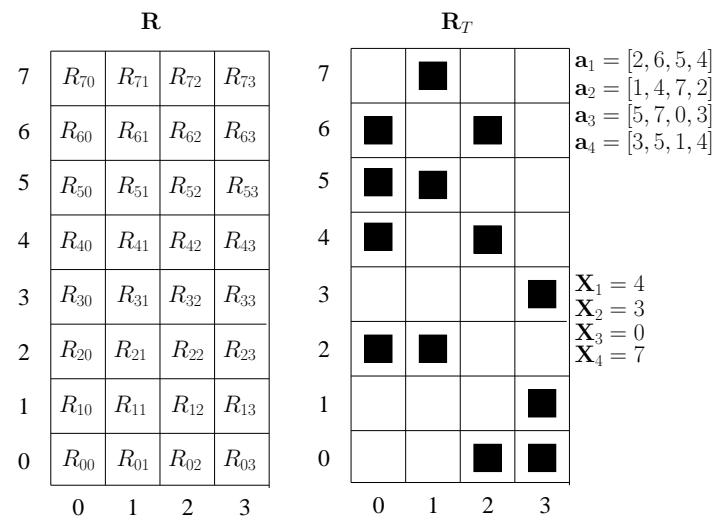

Fig. 2. Time-frequency matrices operated at the relay.

Using $R_{m l}$ for $m=0, \ldots, M-1$ and $l=0, \ldots, L-1$ as entries, the relay can form a $(M \times L)$ TF matrix $\boldsymbol{R}$, as shown in Fig. 2, where $M$ rows correspond to the $M$ frequencies and $L$ columns to the $L$ chips of a symbol. During the broadcasting phase, the relay forwards the information received from the multiple-access phase to the $K$ users by sending them the TF matrix $\boldsymbol{R}$. However, in the TF matrix $\boldsymbol{R}$, the maximum number of entries per column activated during the multiple-access phase is $K$. Hence, in $R$, there are possibly many entries having not been activated by any users and these entries are only constituted by noise and not necessary to be forwarded during the broadcasting phase. Furthermore, forwarding directly the soft information of the entries in $R$ is resource wasteful. For this sake, the relay can carry out certain decision, in order to form a binary TF matrix in the form of $\boldsymbol{R}_{T}$, as shown in Fig. 2. Specifically, in this paper, the threshold-based detection is employed by the relay to 
obtain $\boldsymbol{R}_{T}$. The relay compares each of the entries in $\boldsymbol{R}$, say $R_{m l}$, with a pre-defined threshold $\lambda_{t h}$. If $R_{m l}>\lambda_{t h}$, the corresponding $(m, l)$ th entry in $\boldsymbol{R}_{T}$ is marked (or ' 1 '). Otherwise, the $(m, l)$ th entry in $\boldsymbol{R}_{T}$ is empty (or ' 0 ').

As shown in the TF matrix $\boldsymbol{R}_{T}$, an entry is either marked (' 1 ') or empty ('0'). During the broadcasting phase, the relay forwards the locations of the marked entries to the $K$ users. Therefore, based on $\boldsymbol{R}_{T}$, the TF signal transmitted by the relay during the broadcasting phase can be expressed as

$$
\begin{aligned}
S_{R}(t)=\sqrt{2 P} & \sum_{l=0}^{L-1} \sum_{n=0}^{N_{l}-1} \psi_{T_{h}}\left(t-l T_{h}\right) \\
& \times \exp \left(j 2 \pi \bar{f}_{l}^{(n)} t+\phi_{l}^{(n)}\right)
\end{aligned}
$$

where $N_{l}$ represents the number of marked entries in the $l$ th column of $\boldsymbol{R}_{T}$ and $\left\{\bar{f}_{l}^{(0)}, \bar{f}_{l}^{(1)}, \cdots, \bar{f}_{l}^{\left(N_{l}-1\right)}\right\}$ are the frequency tones corresponding to the marked entries.

Note that, as the above description shows, the relay does not use CSI. It also does not require the knowledge about the FH addresses of the $K$ users. Hence, it has no need or the ability to recover the messages transmitted by the $K$ users. Therefore, the complexity is relatively low for the relay, while the information security is relatively high for the aided users.

Without loss of generality, let us below focus on user 1 and explain how it recovers the messages sent by the other $(K-1)$ users during the multiple-access phase. For this purpose, user 1 utilizes the square-law detection, which is the same as that operated at the relay, to form a TF matrix $\boldsymbol{R}_{1}$ that is also in the form of $\boldsymbol{R}$, as shown in Fig. 2. Based on (5) and following the derivation for (4), we can show that the $(m, l)$ th entry of $R_{1}$ is given by

$$
\begin{aligned}
R_{m l}^{(1)}= & \left|\sum_{n=0}^{N_{l}-1} h_{l}^{(1)} \delta\left(\bar{f}_{l}^{(n)}, f_{m}\right)+N_{m l}^{(1)}\right|^{2}, \\
& m=0,1, \ldots, M-1 ; l=0,1, \ldots, L-1
\end{aligned}
$$

where $N_{m l}^{(1)}$ is a Gaussian noise sample distributed with mean zero and a variance $\sigma_{1}^{2}=L N_{0} / E_{s}$. Furthermore, $R_{m l}^{(1)}$ follows an exponential distribution with a mean of $K_{m l}^{(1)}+\sigma_{1}^{2}$, where $K_{m l}^{(1)}=0$ or 1 , depending on whether the $m$ th frequency tone was transmitted by the relay during the $l$ th chip.

Based on the TF matrix $\boldsymbol{R}_{1}$, the principles of user 1 detecting the other users is now detailed in the next section.

\section{NONCOHERENT DETECTION}

In this section, three types of noncoherent detection schemes are considered, which are the ML-MUD, SML-MUD and MVSUD [13].

\section{A. Maximum Likelihood Detector}

Let $\boldsymbol{s}=\left[X_{1}, X_{2}, \ldots, X_{K}\right]^{T}$ contains the possible $M$-ary symbols transmitted by the $K$ users. Explicitly, when considering the detection at user 1 , its symbol $X_{1}$ is already known. For a given symbol vector $s$ and the set of unique FH addresses $\left\{\boldsymbol{a}_{k}\right\}$ of $K$ users, the probability density function (PDF) of the received TF matrix $\boldsymbol{R}_{1}$ at user 1 can be denoted as $f\left(\boldsymbol{R}_{1} \mid \boldsymbol{s},\left\{\boldsymbol{a}_{k}\right\}\right)$. Under the assumption that the $M L$ entries in $\boldsymbol{R}_{1}$ are mutually independent, this PDF can be written as

$$
f\left(\boldsymbol{R}_{1} \mid \boldsymbol{s},\left\{\boldsymbol{a}_{k}\right\}\right)=\prod_{m=0}^{M-1} \prod_{l=0}^{L-1} f\left(R_{m l}^{(1)} \mid \boldsymbol{s},\left\{\boldsymbol{a}_{k}\right\}\right)
$$

where $f\left(R_{q l}^{(1)} \mid \boldsymbol{s},\left\{\boldsymbol{a}_{k}\right\}\right)$ denotes the PDF of $R_{q l}^{(1)}$, when given $\boldsymbol{s}$ and the FH addresses $\left\{\boldsymbol{a}_{k}\right\}$.

According to the principles of ML-MUD, the optimum solution is obtained by searching the signal space and finding the symbol vector $\hat{s}$ that maximizes the likelihood function $f\left(\boldsymbol{R}_{1} \mid \boldsymbol{s},\left\{\boldsymbol{a}_{k}\right\}\right)$ or the log-likelihood function $\ln f\left(\boldsymbol{R}_{1} \mid \boldsymbol{s},\left\{\boldsymbol{a}_{k}\right\}\right)$, which can be stated as

$$
\begin{aligned}
\hat{\boldsymbol{s}} & =\arg \max _{\boldsymbol{s} \in \mathcal{X}^{K}}\left\{\prod_{m=0}^{M-1} \prod_{l=0}^{L-1} f\left(R_{m l}^{(1)} \mid \boldsymbol{s},\left\{\boldsymbol{a}_{k}\right\}\right)\right\} \\
& =\arg \max _{\boldsymbol{s} \in \mathcal{X}^{K}}\left\{\sum_{m=0}^{M-1} \sum_{l=0}^{L-1} \ln f\left(R_{m l}^{(1)} \mid \boldsymbol{s},\left\{\boldsymbol{a}_{k}\right\}\right)\right\},
\end{aligned}
$$

where the space $\mathcal{X}=\{0,1, \ldots, M-1\}$ contains the possible $M$-ary symbols transmitted.

In the above equations, the PDF of $f\left(R_{m l}^{(1)} \mid \boldsymbol{s},\left\{\boldsymbol{a}_{k}\right\}\right)$ can be expressed as

$$
\begin{gathered}
f\left(R_{m l}^{(1)} \mid \boldsymbol{s},\left\{\boldsymbol{a}_{k}\right\}\right)=P\left(R_{T}^{(m, l)}=1 \mid \boldsymbol{s},\left\{\boldsymbol{a}_{k}\right\}\right) f\left(R_{m l}^{(1)} \mid R_{T}^{(m, l)}=1\right) \\
+P\left(R_{T}^{(m, l)}=0 \mid \boldsymbol{s},\left\{\boldsymbol{a}_{k}\right\}\right) f\left(R_{m l}^{(1)} \mid R_{T}^{(m, l)}=0\right), \quad(10)
\end{gathered}
$$

where $P\left(R_{T}^{(m, l)}=i \mid \boldsymbol{s},\left\{\boldsymbol{a}_{k}\right\}\right), i=1,0$, is the probability that the $(m, l)$ th entry of $\boldsymbol{R}_{T}$ is marked ( $i=1$ ) or empty ( $i=0$ ), when given the symbol vector $s$ and the set of FH addresses $\left\{\boldsymbol{a}_{k}\right\}$ of the $K$ users, while $f\left(R_{m l}^{(1)} \mid R_{T}^{(m, l)}=i\right)$ represents the PDF of $R_{m l}^{(1)}$ conditioned on $R_{T}^{(m, l)}=i$. Specifically, when a threshold $\lambda_{t h}$ is used, we have for $m=0,1, \ldots, M-1$ and $l=0,1, \ldots, L-1$

$$
\begin{gathered}
P\left(R_{T}^{(m, l)}=1 \mid \boldsymbol{s},\left\{\boldsymbol{a}_{k}\right\}\right)=\exp \left(-\frac{\lambda_{t h}}{K_{m l}+\sigma_{r}^{2}}\right) \\
P\left(R_{T}^{(m, l)}=0 \mid \boldsymbol{s},\left\{\boldsymbol{a}_{k}\right\}\right)=1-\exp \left(-\frac{\lambda_{t h}}{K_{m l}+\sigma_{r}^{2}}\right) \\
f\left(R_{m l}^{(1)} \mid R_{T}^{(m, l)}=1\right)=\frac{1}{1+\sigma_{1}^{2}} \exp \left(-\frac{R_{m l}^{(1)}}{1+\sigma_{1}^{2}}\right) \\
f\left(R_{m l}^{(1)} \mid R_{T}^{(m, l)}=0\right)=\frac{1}{\sigma_{1}^{2}} \exp \left(-\frac{R_{m l}^{(1)}}{\sigma_{1}^{2}}\right) .
\end{gathered}
$$

Upon applying the above equations and (10) into (8) or (9), we can obtain the optimum solution by searching a space of size $M^{(K-1)}$.

Despite achieving the optimum performance, the complexity of the ML-MUD is very high, which grows exponentially with the number of users invoked. Furthermore, the ML-MUD is sensitive to the non-ideal conditions, such as a non-optimum threshold used by the relay. Therefore, we below consider two other detectors having significantly lower complexity than the ML-MUD.

\section{B. Majority Vote Based Detector}

The principles of the MV-SUD can be found in Appendix 7.A of [13], which can be briefly summarized as follows. From the received observation matrix $\boldsymbol{R}_{1}$, user 1 first forms a harddecision TF matrix $\boldsymbol{R}_{T}^{(1)}$ in the form of $\boldsymbol{R}_{T}$, as shown in Fig. 2, by comparing each of its entries with a threshold, say, $\lambda_{t h}^{(1)}$. If the entry's value is higher than $\lambda_{t h}^{(1)}$, the corresponding TF element in $\boldsymbol{R}_{T}^{(1)}$ is marked. Otherwise, it is left empty. After 
obtaining $\boldsymbol{R}_{T}^{(1)}$, user 1 uses, respectively, the FH addresses of user $2,3, \ldots, K$ to de-hop it, obtaining $\boldsymbol{D}_{2}^{(1)}, \boldsymbol{D}_{3}^{(1)}, \ldots, \boldsymbol{D}_{K}^{(1)}$ for detection of the symbols transmitted by user $2,3, \ldots, K$. Specifically, based on $\boldsymbol{D}_{k}^{(1)}, k=2, \ldots, K$, user 1 makes a decision in favour of the $M$-ary symbol $\hat{X}_{k}$ corresponding to the specific row that has the highest number of marked entries. This symbol represents the estimate to the symbol $X_{k}$ transmitted by user $k$. In the case that there are more than one rows having the highest number of marked entries, user 1 then randomly selects one and uses it as the estimate of $X_{k}$.

\section{Sub-optimum Maximum Likelihood Detector}

The principles of the SML-MUD is the same as that of the ML-MUD, except that the SML-MUD works with a space whose size is significantly smaller than $M^{K-1}$, the size of the ML-MUD's search space [13]. In order to obtain such a sub-space that does not result in significant performance penalty, as shown in Section 7.7 of [13], user 1 first dehops $\boldsymbol{R}_{T}^{(1)}$ using the FH addresses of user $2,3, \ldots, K$ to obtain $\boldsymbol{D}_{2}^{(1)}, \boldsymbol{D}_{3}^{(1)}, \ldots, \boldsymbol{D}_{K}^{(1)}$. Based on the de-hopped matrices $\boldsymbol{D}_{2}^{(1)}, \boldsymbol{D}_{3}^{(1)}, \ldots, \boldsymbol{D}_{K}^{(1)}$, the candidate symbols for user $2,3, \ldots, K$ are formed. Specifically, depending on the complexity affordable, the symbols corresponding to the complete rows, to the rows having $(L-1),(L-2)$, etc., entries in $\boldsymbol{D}_{2}^{(1)}, \boldsymbol{D}_{3}^{(1)}, \ldots, \boldsymbol{D}_{K}^{(1)}$ may be taken as the candidate symbols transmitted by user $2,3, \ldots, K$. Let $\mathcal{X}_{k}, k=2, \ldots, K$, collects the candidate symbols of user $k$. Then, for the SMLMUD, the estimate to the transmitted symbol vector $s$ can be found according to the optimization problem

$$
\begin{aligned}
\hat{\boldsymbol{s}} & =\arg \max _{\left\{X_{k} \in \mathcal{X}_{k}\right\}_{k=2}^{K}}\left\{\prod_{m=0}^{M-1} \prod_{l=0}^{L-1} f\left(R_{m l}^{(1)} \mid \boldsymbol{s},\left\{\boldsymbol{a}_{k}\right\}\right)\right\} \\
& =\arg \max _{\left\{X_{k} \in \mathcal{X}_{k}\right\}_{k=2}^{K}}\left\{\sum_{m=0}^{M-1} \sum_{l=0}^{L-1} \ln f\left(R_{m l}^{(1)} \mid \boldsymbol{s},\left\{\boldsymbol{a}_{k}\right\}\right)\right\},
\end{aligned}
$$

Below we provide a range of results to illustrate the achievable error performance of the FFH/MFSK MWR systems, when the above-mentioned three types detection schemes are invoked.

\section{Simulation Results}

In this section, the BER performance of the FFH/MFSK MWR systems is investigated by simulations, when assuming communications over Rayleigh fading channels. Some of the parameters used in our simulations are depicted associated with the figures. In the examples considered, we assumed that the users employed the optimum FH addresses [10] and that all the users transmit with the same power. The SNR per bit shown in the figures is the average SNR per bit, given by $\gamma_{b}=E_{b} / N_{0}=E_{s} /\left(b N_{0}\right)$, where $b=\log _{2} M$ represents the number of bits per MFSK symbol. From the above settings, we are implied that the energy consumed at the relay is $K E_{s}$ per symbol duration, which grows lineally with the number of users involved. Note that, in practice, in an infrastructurebased network, high-power nodes may be deployed as the relays. By contrast, in an infrastructure-less network, such as in an ad-hoc network, nodes may be chosen as the relay nodes alternatively, or chosen as the relay nodes based on certain optimization criteria, such as, maximum network lifetime, maximum reliability, etc. Note furthermore that, for the SMLMUD, in our simulations, the rows having either $L$ or $(L-1)$ entries in the TF matrices $\left\{\boldsymbol{D}_{i}\right\}$ were chosen to generate candidate symbols for finding the final solution.

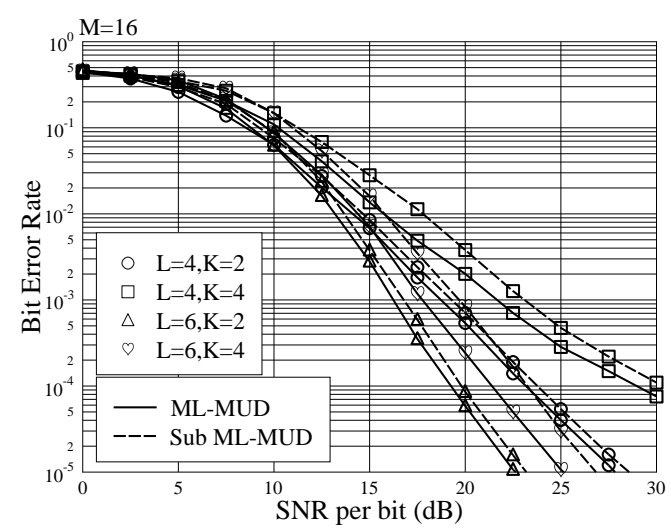

Fig. 3. BER performance of the FFH/MFSK MWR systems employing ML-MUD.

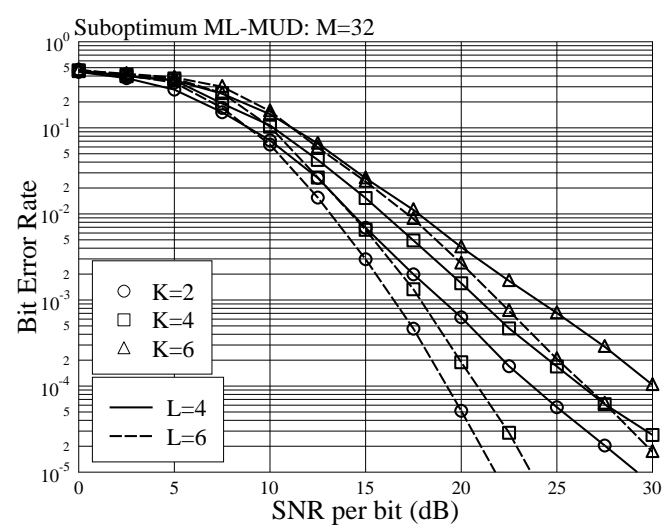

Fig. 4. BER performance of the FFH/MFSK MWR systems employing SML-MUD.

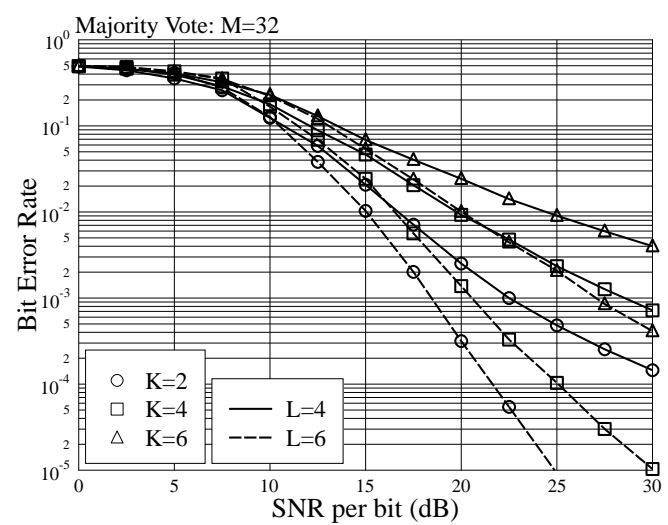

Fig. 5. BER performance of the FFH/MFSK MWR systems employing MV-SUD.

Figs. 3, 4 and 5 depict the BER performance of the FFH/MFSK MWR systems, when the ML-MUD, supoptimum ML-MUD and MV-SUD are employed, respectively. In addition to the parameters shown in the figures, in our simulations, the thresholds used by the relay and the users were set in such a way that the false-alarm probability was lower than $10^{-4}$ while minimizing the miss probability. From 
the results of Figs. 3 - 5, we can derive the following observations. First, for a give value of $K$, the BER performance improves, as the value of $L$ increases. Second, for a given value of $L$, the BER performance deteriorates, as the number of users $K$ increases. Third, when comparing the results of the ML-MUD with that of the SML-MUD, as shown in Fig. 3, the ML-MUD outperforms slightly the SML-MUD. However, due to the threshold detection carried out at the relay and that the threshold may not be optimum, error-floors start appearing for the case $L=4, K=4$, even for the ML-MUD. Finally, the MV-SUD yields the worst BER performance among the three detectors considered. However, we should value that the MV-SUD has the lowest complexity, which does not mind at all the statistics of channels and transmitted signals.

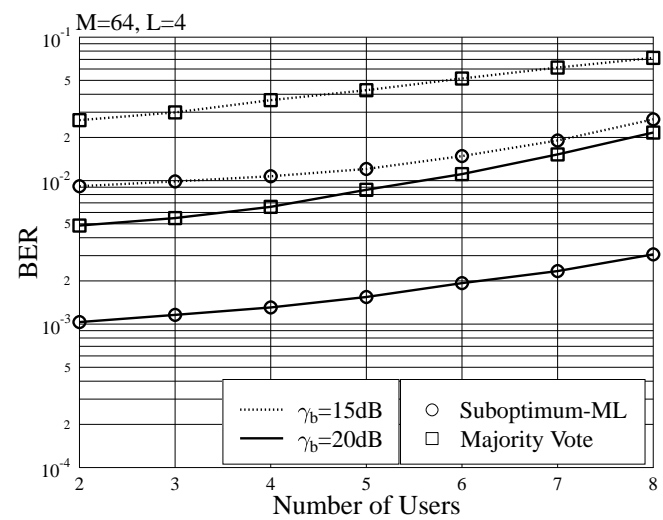

Fig. 6. BER versus the number of user $K$ for the FFH/MFSK MWR systems employing either SML-MUD or MV-SUD.

Fig. 6 shows the BER performance of the FFH/MFSK MWR systems against the number of user $K$. Again, the thresholds were set to minimize the miss probability, when given the false-alarm probability lower than $10^{-4}$. Explicitly, the BER for both the detectors slightly increases, as the number of users involved increases. The SML-MUD outperforms the MV-SUD in terms of the BER performance.

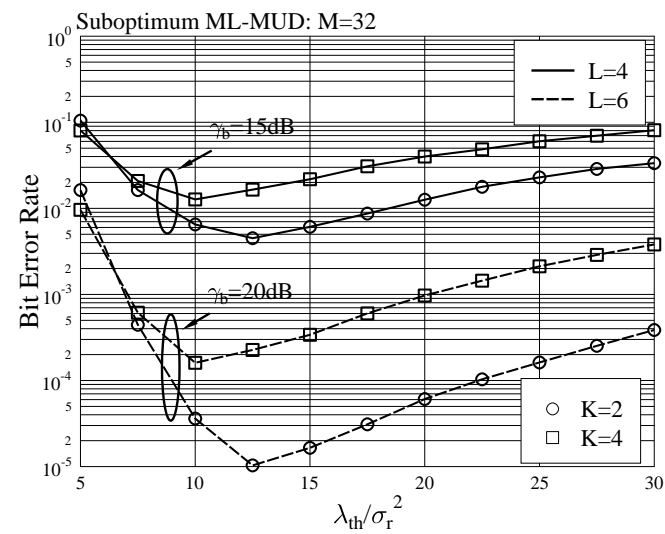

Fig. 7. BER versus threshold performance for the FFH/MFSK MWR systems employing SML-MUD.

Finally, Fig. 7 investigates the impact of the threshold used by the relay on the BER performance of the FFH/MFSK MWR systems employing the SML-MUD. Explicitly, for any given values of $K, L$ and SNR, there exists an optimum threshold, which makes the FFH/MFSK MWR systems achieve the best BER performance.

\section{CONCLUSiOns}

In order to achieve high-efficiency information exchange among a group of users, in this contribution, we have proposed and studied a FFH/MFSK MWR scheme, which implements the information exchange using two time-slots regardless of the number of users invoked. The relay and final detection strategies have been designed. The error performance of the FFH/MFSK MWR systems has been investigated by simulations, when the ML-MUD, SML-MUD or the MV-SUD is employed. It can be shown that the FFH/MFSK MWR scheme can facilitate information exchange among a group of users. It can be designed to make efficient use of the frequency resources. It can naturally exploit the frequencyselective fading to achieve frequency diversity. Furthermore, the FFH/MFSK MWR systems can be implemented with lowcomplexity noncoherent techniques.

\section{ACKNOWLEDGEMENT}

The work of the first and third authors was supported by the Joint State Key Program of National Natural Science Fundation of China (Grant No: 60830001).

\section{REFERENCES}

[1] E. van der Meulen, "A survey of multi-way channels in information theory: 1961-1976," IEEE Trans. on Infor. Theory, vol. 23, no. 1, pp. - 37, Jan 1977.

[2] D. Gunduz, A. Yener, A. Goldsmith, and H. Poor, "The multi-way relay channel," IEEE Inter. Sym. on Infor. Theory, pp. 339 - 343, July 2009.

[3] A. U. T. Amah and A. Klein, "A transceiver strategy for regenerative multi-antenna multi-way relaying," IEEE Inter. Workshop on Comput. Adv in Multi-Sensor Adapt. Proc. (CAMSAP), pp. 352 -355, Dec. 2009.

[4] B. Rankov and A. Wittneben, "Achievable rate regions for the two-way relay channel," IEEE Inter. Symp. on Infor. Theory, pp. $1668-1672$, July 2006.

[5] D. Chen and J. N. Laneman, "Modulation and demodulation for cooperative diversity in wireless systems," IEEE Trans. on Wireless Comm. vol. 5, no. 7, pp. 1785-1794, July 2006.

[6] R. Annavajjala, P. C. Cosman, and L. B. Milstein, "On the performance of optimum noncoherent amplify-and-forward reception for cooperative diversity," IEEE MILCOM'2005, pp. 3280-3288, Vol. 5, Oct. 2005.

[7] M. R. Souryal, "Non-coherent amplify-and-forward generalized likelihood ratio test receiver," IEEE GLOBECOM'2008, pp. 1-6, Dec. 2008.

[8] M. R. Souryal and H. You, "Diversity performance of a practical noncoherent detect-and-forward receiver," IEEE GLOBECOM'2008, pp. 16, Dec. 2008.

[9] D. J. Goodman, P. S. Henry, and V. K. Prabhu, "Frequency-hopped multilevel FSK for mobile radio," Bell Syst. Tech. J., vol. 59, no. 7, pp. 1257-1275, Sep 1980.

[10] G. Einarsson, "Address assignment for a time-frequency-coded, spread spectrum systems," Bell Syst. Tech. J., vol. 59, no. 7, pp. 1241-1255, Sep 1980.

[11] C. Hung and Y. Su, "Diversity combining considerations for incoherent frequency hopping multiple access systems," IEEE J. on Select. Areas in Commun., vol. 13, no. 2, pp. 333 -344, Feb. 1995.

[12] Y. Su, Y.-S. Shen, and C.-Y. Hsiao, "On the detection of a class of fast frequency-hopped multiple access signals," IEEE J. on Select. Areas in Commun., vol. 19, no. 11 , pp. 2151 -2164, Nov. 2001

[13] L.-L. Yang, Multicarrier Communications. John Wiley \& Sons, Chichester, UK, 2009

[14] K. Halford and M. Brandt-Pearce, "Multistage multiuser detection for FHMA," IEEE Trans. on Commun., vol. 48, no. 9, pp. 1550-1562, Sept. 2000.

[15] J. G. Proakis, Digital Communications, 3rd ed. McGraw Hill, 1995. 